\title{
New Algorithm of Seed Finding for Track Reconstruction
}

\author{
Dmitry Baranov ${ }^{1, a}$, Sergei Merts ${ }^{1, b}$, Gennady Ososkov ${ }^{1, \mathrm{c}}$, and Oleg Rogachevsky ${ }^{1, \mathrm{~d}}$ \\ ${ }^{1}$ Joint Institute for Nuclear Research, 6 Joliot Curie St., Dubna, Moscow region, 141980 Russia
}

\begin{abstract}
Event reconstruction is a fundamental problem in the high energy physics experiments. It consists of track finding and track fitting procedures in the experiment tracking detectors. This requires a tremendous search of detector responses belonging to each track aimed at obtaining so-called "seeds", i.e. initial approximations of track parameters of charged particles. In the paper we propose a new algorithm of the seedfinding procedure for the BM@N experiment.
\end{abstract}

\section{Introduction}

BM@N (Baryonic Matter at Nuclotron) is a fixed target experiment at the JINR Nuclotron for the study of $A+A$ collisions by measuring a variety of observables [1]. The proposed set-up for this experiment is shown in figure 1 .

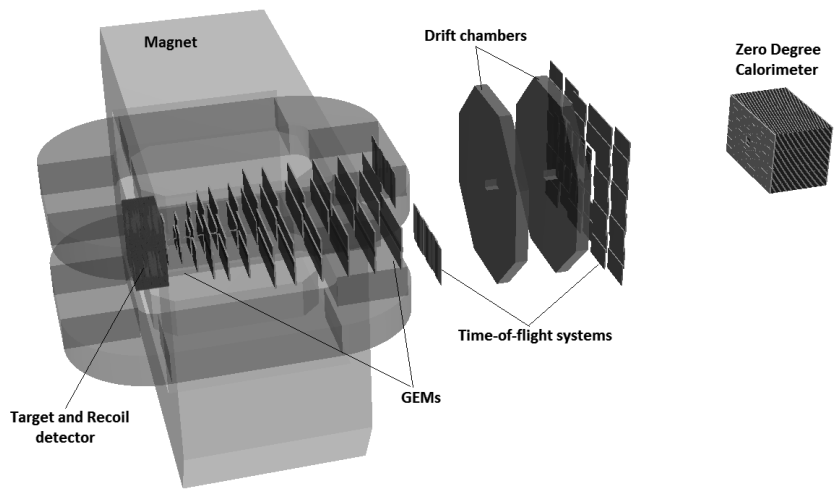

Figure 1. Set-up of the BM@N experiment

It combines high precision track measurements with time-of-flight information for particle identification and total energy measurements for event characterization. The charged particles will be

\footnotetext{
ae-mail: dmitriybaranof@gmail.com

be-mail: Sergey.Merts@gmail.com

ce-mail: gososkov@gmail.com

de-mail: rogachevsky@jinr.ru
} 
measured with the set of GEM (Gaseous Electron Multipliers) detectors located downstream of the target inside the analyzing magnet of $0.8 \mathrm{~T}$ and drift chambers situated outside the magnetic field. Design parameters of the time-of-flight detectors based on multigap Resistive Plate Chambers (mRPC) with a strip read-out allow efficient discrimination between particle species with momentum up to a few $\mathrm{GeV} / \mathrm{c}$. The Zero degree calorimeter (ZDC) is designed for the collision centrality analysis by measuring the energy of forward going particles. The Recoil detector, partially covering the backward hemisphere $(-1.0<\eta<1.2)$ near the target, is planned for the independent analysis of the collision centrality by the measurement of the energy of the target fragments.

The event reconstruction for the BM@N experiment consists in finding track seeds in the GEM detectors and then extrapolate them with the Kalman filter [2] to other detectors. As one can see from the left image of the figure 3, the seed finding problem is really hard and time consuming on any projection due to overcrowding the area of search by hits ${ }^{1}$. To find a seed from the set of hits registered by a tracking detector one should classify somehow those hits by their closeness to particle trajectories. There are several methods used to achieve that, as Hough transform, elastic tracking, cellular automaton etc, with their advantages and shortcomings [3, 4]. We propose a new transformation of the coordinate system which results in a transformed space, where hits corresponding to a same track are grouped more compactly.

\section{Track parameters and coordinate transformation}

Let us consider a parametric helix equation that describes a charged particle trajectory in a homogeneous magnetic field (the direction of the magnetic field is parallel to the $y$ axis):

$$
x=r \cos t, \quad y=c t, \quad z=r \sin t,
$$

where $r$ is the radius of the helix and $c$ is a constant giving the vertical separation of the helix loops.

Let us make a substitution

$$
\tilde{x}=\frac{x}{R}, \quad \tilde{y}=\frac{y}{R},
$$

where $R=\sqrt{x^{2}+y^{2}+z^{2}}=\sqrt{r^{2}+c^{2} t^{2}}$.

Using (1) and (2) one can obtain the expressions:

$$
\tilde{x}=\frac{r \cos t}{\sqrt{r^{2}+c^{2} t^{2}}}, \quad \tilde{y}=\frac{c t}{\sqrt{r^{2}+c^{2} t^{2}}} .
$$

Getting $t$ from the second equation of (3) and replacing it in the first equation of (3) we obtain a relationship between $\tilde{y}$ and $\tilde{x}$ :

$$
\tilde{x}=\sqrt{1-\tilde{y}^{2}} \cdot \cos \left[\frac{r \tilde{y}}{c \sqrt{1-\tilde{y}^{2}}}\right] .
$$

The parameter $k=r / c$ is directly related to the polar angle and the momentum of the track. For tracks to be reconstructed in the BM@N experiment the polar angle is less than $30^{\circ}$ and the momentum more than $100 \mathrm{MeV} / \mathrm{c}$. The emerging $k$ value is $k>100$ and the helix (1) is transformed to the fast oscillating curve with almost horizontal waves, as shown in the figure 2.

Tracks in the BM@N experiment take only part of the helix and their transformation follows to central, approximately horizontal, fragments of the waves shown in figure 2. This can be observed in the figure 3 where simulated hits for $\mathrm{Au}-\mathrm{Au}$ collision with $E_{\mathrm{lab}}=4 \mathrm{AGeV}$ and their images after coordinate transformation $(\{x, y\} \longmapsto\{x / R, y / R\})$ are presented.

\footnotetext{
${ }^{1}$ hit is an reconstructed spatial point of crossing track and subdetector system.
} 

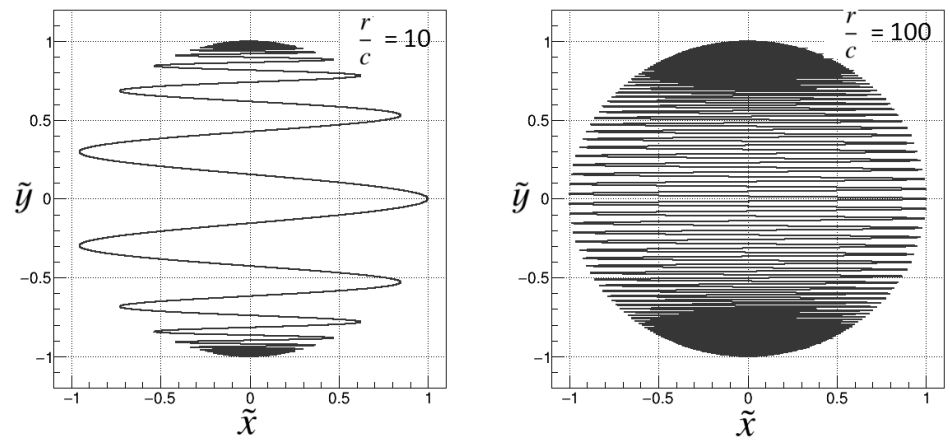

Figure 2. Helix in $\{\tilde{x}, \tilde{y}\}$ space for $r / c=10$ (left) and $r / c=100$ (right)
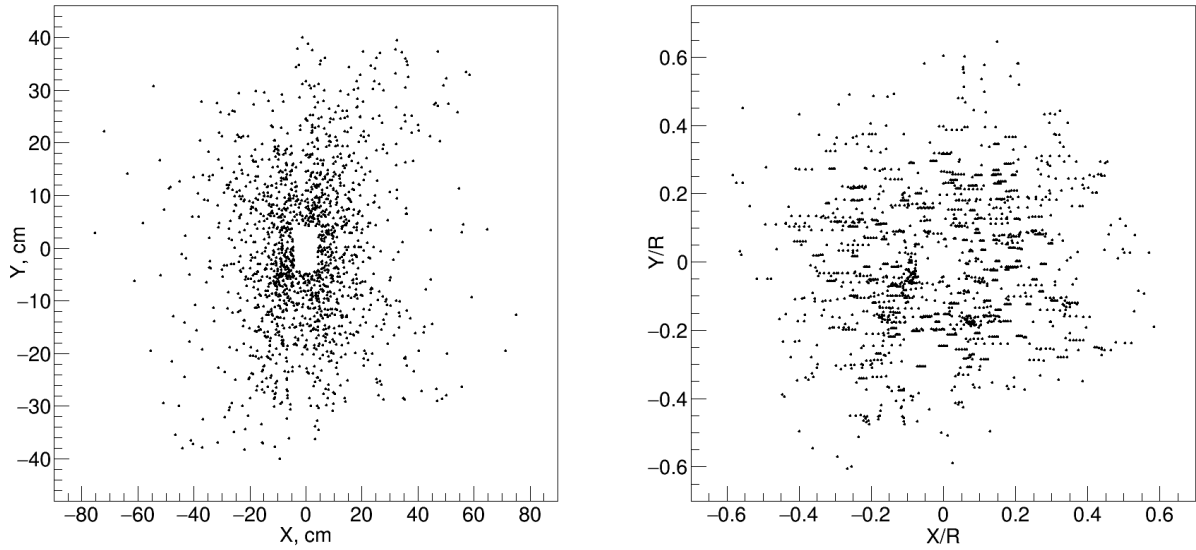

Figure 3. Left: $\mathrm{XY}$ view of a simulation for the first 5 GEM-stations of the BM@N facility; Right: representation of the same tracks after using transformation $\{x, y\} \longmapsto\{x / R, y / R\}$

This algorithm for track-candidate searching with the fast horizontal histogramming was implemented in BmnRoot [5] framework. The distributions of the efficiency and the percent of the ghosttracks for the ideal case are shown in figure 4. The ideal case is the simulation with a pixel readout, without taking into account the strip readout of the GEM detector.

\section{Comparison of the complexity of "standard" and proposed algorithms}

Complexity of the "standard" algorithm

1. A combinatorics of all hits belonging to a station requires $O(n)$ operations.

2. To collect at least three hits to start the Kalman filter, $O\left(n^{3}\right)$ operations are required.

3. Parameters of the found track-candidate should be estimated. It requires $O(1)$ operations. 


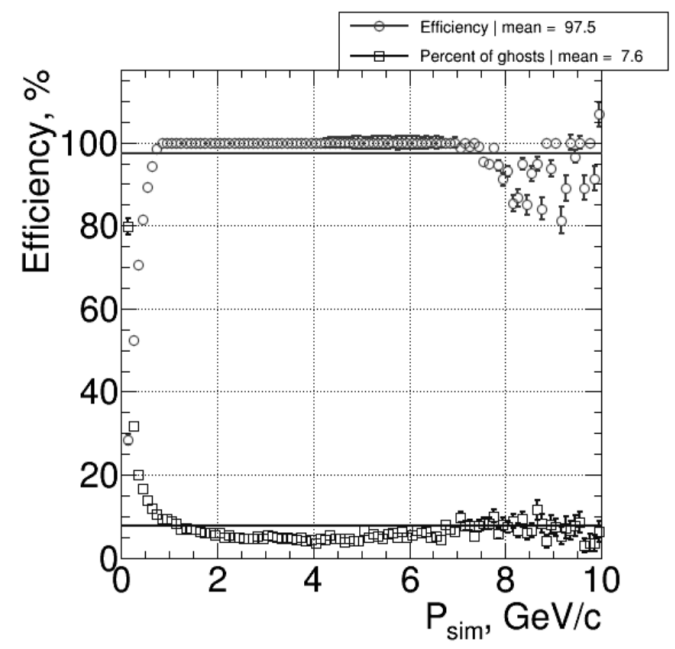

Figure 4. BM@N track reconstruction efficiency for transformed data in an ideal case.

4. As a result, the complexity of the "standard" algorithm equals $T=O(n) \cdot O\left(n^{3}\right) \cdot O(1)=O\left(n^{3}\right)$ operations. Complexity of the present algorithm

1. A combinatorics of all hits belonging to the first station requires $O(n)$ operations.

2. The search procedure using a "corridor" of the fixed length and width should be performed on the selected hit. It requires $O(1)$ operations.

3. Parameters of the found track-candidate should be estimated. It requires $O(1)$ operations.

4. Finally, the complexity of the present algorithm equals $T=O(n) \cdot O(1) \cdot O(1)=O(n)$ operations.

\section{Fake hits rejection}

The main shortcoming of the BM@N data acquisition from the GEM detector is the appearance of fake hits caused by extra spurious strip crossings. For $n$ real hits and orthogonal strips the number of fakes is $n^{2}-n$. One way to decrease the fake number is to rotate the strips of one layer at a small angle $\left(5^{\circ}-15^{\circ}\right)$ with respect to another layer. But still the number of fakes is about $\left(n^{2}-n\right) \cdot \sin (\alpha)$, where $\alpha$ is the angle between strips.

In the current work we propose two methods for fake hits rejection.

1. Using of particle energy losses

Because the charged particles have different energy losses on different strips, but energy losses of the same particle on strips of the upper and lower layers are almost equal.

The main idea of fake rejection is the next: the real hit is formed only when two strips with equal charge are crossed. All other crossings which belong to those strips are considered as fakes (see figure 5).

Point $r_{i j}$ is accepted as hit, if the absolute value of the relative differences of energy losses in two crossing strips do not exceed a given threshold. 

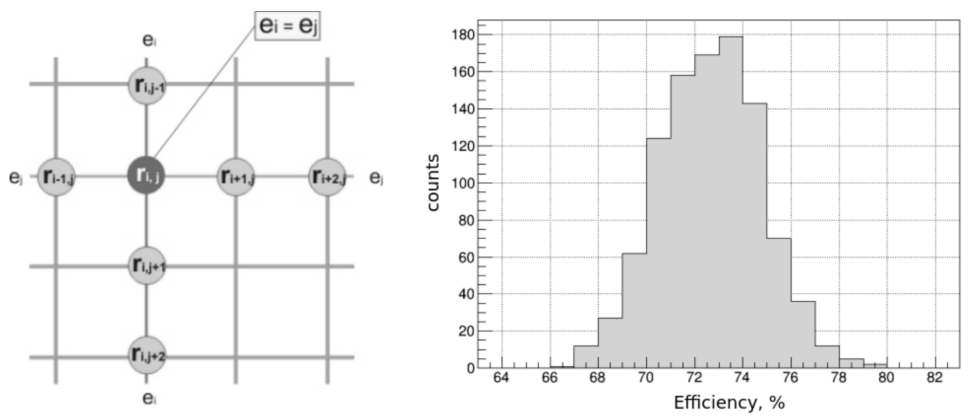

Figure 5. Left: scheme of fakes finding ( $e_{i}-$ energy of $i$-strip, $e_{j}$ - energy of $j$-strip). Right: efficiency of fakes rejection.

Algorithm efficiency evaluated by comparison of hit identifiers obtained by the algorithm with known Monte Carlo identifiers. The algorithm is a two-steps one: at the first step it is determined whether two strips crossing is a hit. Only if it is true then the second step is accomplished when all fakes are searched lying on the corresponding strips. Therefore the efficiency directly depends on the number of the found hits (see figure 5).

2. Using of potential approach for data contaminated by fakes

The fake contamination of data for the BM@N GEM detector brought us serious obstacles for carrying out our algorithm for track candidate search, as it is seen on the left panel of figure 6 , where an UrQMD Au-Au event for the only last 5 stations even after $\{x / R, y / R\}$ transform and fake rejection is presented.
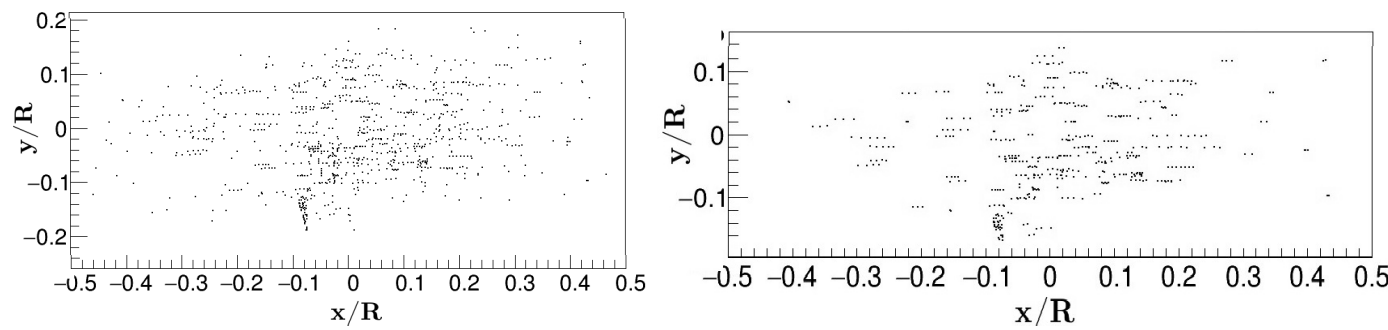

Figure 6. UrQMD Au-Au events in $\{x / R, y / R\}$ space for last 5 GEM-stations. Left: before Lorenz potential applying. Right: after applying the potential

So we should enhance the influence of the transformed track properties as closeness and horizontality. It can be done by attributing to every hit such a potential that would emphasize only the useful track properties. The Lorenz potential was chosen with horizontal half-width $\sigma_{x}$ and ordinate width $\sigma_{y}$ :

$$
V(x, y)=\frac{1}{1+\frac{\left(x-x_{i}\right)^{2}}{\sigma_{x}^{2}}+\frac{\left(y-y_{i}\right)^{2}}{\sigma_{y}^{2}}}
$$




\section{EPJ Web of Conferences}

Applying and then summarizing this potential to every hit on this plane with properly chosen $\sigma_{x}$ and $\sigma_{y}$, we obtain potentials of close hits form a peak, the amplitude of which depends on the corresponding particle momentum. On the right panel of the figure 6 the result of applying the Lorenz potential with $\sigma_{x}=0.028, \sigma_{y}=0.00075$ and amplitude cut equal 1.5 demonstrates the significant improvement of transformed data to which now the algorithm for track candidate search is applicable.

Although a direct potential application is time consuming, their compactness allows to reduce the computational procedure to a faster sliding window liable to be parallelized.

\section{Conclusion}

A new algorithm of searching for track-candidates has been proposed and tested for BM@N data. The developed algorithm allows considerable decrease of combinatorics. A high speed of execution of the algorithm has been achieved. A new approach applying Lorenz potential to improve track-candidate search in cases of high multiplicity is proposed. The study of fake hits appearance was fulfilled and effective procedures for the fake rejection were proposed.

\section{References}

[1]BM@N Conceptual Design Report (BM@N collaboration), http://nica.jinr.ru/files/BM@N/ BMN_CDR.pdf

[2] R. Fruhwirth, Nucl. Instrum. Meth. A 262, 444 (1987)

[3] R. Fruhwirth et al., Data Analysis Techniques for High-Energy Physics, 2nd ed. Cambridge Univ. Press (2000)

[4] G. A. Ososkov, A. Polyansky, I. V. Puzynin, Phys.Part.Nucl. 33 3, 676-741 (2002) [in Russian]

[5] BmnRoot framework, http://mpd.jinr.ru 